\title{
Computed Tomography of the Neck, Chest, Abdomen and Pelvis without Contrast
}

National Cancer Institute

\section{Source}

National Cancer Institute. Computed Tomography of the Neck, Chest, Abdomen and

Pelvis without Contrast. NCI Thesaurus. Code C137903.

Computed tomography of the neck, chest, abdomen and pelvis without the use of a contrast agent. 